\title{
FONDEMENTS ET CONTENU DE L'ASSISTANCE ELECTORALE DE LA MONUSCO : NECESSITE DE CERTIFICATION INTERNATIONALE DU PROCESSUS
}

\author{
Par MUKIRAMFI SAMBA*
}

\begin{abstract}
Résumé
L'implication de l'ONU dans le processus électoral post-conflit en République Démocratique du Congo est considérée comme un élément pour la consolidation de la paix, qui aiderait à renverser toutes tendances vers la concentration du pouvoir et l'amenuisement de l'espace politique, ainsi qu'à réduire les sentiments sécessionnistes grandissant dans certaines régions du pays. Or, la réalité du processus électoral, du moins concernant la frange présidentielle et législative du 28 novembre 2011, a montré des insuffisances du mandat actuel de la MONUSCO en la matière, limité à l'appui technique et logistique à la CENI et au monitoring des violations des droits de l'homme en rapport avec le processus électoral, au point de créer des polémiques sur la question. Tout en s'interrogeant sur la nécessité de rationalisation du mandat actuel jugé insuffisant, cet article tend à démontrer le bien-fondé de son élargissement par l'octroi d'un pouvoir de certification du processus aux Nations Unies et ce, au regard de la pratique récente des Nations Unies notamment en Côte d'Ivoire, en tenant certes compte des réalités propres de la République Démocratique du Congo.
\end{abstract}

\section{Introduction}

En plein débat suscité par le Gouvernement congolais sur le retrait de la Mission de l'Organisation des Nations Unies en République démocratique du Congo « MONUC », ${ }^{1}$ le Conseil de sécurité, par la résolution 1925 (2010) adoptée le 28 mai 2010, reconnaissant les progrès réalisés en République démocratique du Congo, compte tenu des défis que le pays a eu à surmonter ces 15 dernières années, décidera que la Mission s'appellerait la Mission de l'Organisation des Nations Unies pour la stabilisation en République démocratique du Congo, MONUSCO en sigle, en remplacement de la MONUC. ${ }^{2}$ Il a estimé qu'il était nécessaire de donner une nouvelle orientation au mandat de la Mission des Nations Unies

* Chef de travaux et doctorant à la Faculté de Droit de l'Université de Kinshasa.

1 Après plus d'une décennie de présence des Nations Unies en RDC sous la forme d'opération de maintien de la paix, la mission confiée à la MONUC avait évolué en fonction des événements. Créée en vue de la mise en œuvre de l'Accord de Lusaka du 10 juillet 1999, elle avait reçu et accompli des tâches énormes partant de l'observation du cessez-le-feu, à la surveillance du désengagement des troupes vers le rétablissement, l'imposition et la consolidation de la paix dans ce pays.

2 Résolution 1925 (2010) du 28 mai 2010, § $1^{\text {er }}$. 
dans le pays, en accordant une importance particulièrement accrue à la consolidation ${ }^{3}$ de la paix pour raffermir et faire avancer la stabilisation du pays, ${ }^{4}$ avec entre autres la possibilité d'apporter au Gouvernement de la RDC un soutien technique et logistique pour l'organisation des élections nationales et locales, à la demande expresse du Gouvernement. ${ }^{5}$

Ces élections libres et équitables sont reconnues ici comme un élément pour la consolidation de la paix, puis que leur tenue aiderait à renverser toutes tendances vers la concentration du pouvoir et l'amenuisement de l'espace politique, ainsi qu'à réduire les sentiments sécessionnistes grandissant dans certaines régions du pays. Or, la réalité du processus électoral, du moins concernant la frange présidentielle et législative du 28 novembre 2011, a montré des insuffisances de ce mandat au point de créer des polémiques sur la question. On a ainsi assisté à une élection émaillée de contestations des résultats, de violences, de violations de libertés et droits fondamentaux liées au processus, constatées et rapportées par les observateurs sur le terrain. ${ }^{6}$ La communauté internationale au travers de la MONUSCO n'a pas manqué encore une fois d'être visée par des critiques.

Il s'agit ici de s'interroger sur l'adéquation entre ce qui fonde le Conseil de sécurité à conférer à cette mission un tel mandat d'assistance électorale et sa consistance-même, étant entendu que cette assistance électorale apportée par l'O.N.U. aux Etats est une pratique qui

3 La consolidation de la paix comprend des mesures ciblées visant à réduire les risques de reprise d'un conflit et de jeter les bases d'un développement durable, notamment à travers le renforcement des capacités nationales en matière de gestion des conflits à tous les niveaux. Les activités de consolidation de la paix visent les causes structurelles d'un conflit armé dans une approche globale. C'est justement ce que dit le Secrétaire général des Nations Unies pour qui la consolidation de la paix en RDC « consiste, dans un pays au sortir d'un conflit, à rétablir l'autorité de l'Etat, à relancer le dialogue entre les pouvoirs publics et la société civile, à permettre à celle-ci de se régénérer et à établir les assises institutionnels du développement économique et social » (Rapport du Secrétaire général sur l'activité de la MONUC, 2006, § 18). Ainsi pour consolider les acquis de la paix, le Conseil de sécurité a chargé la MONUSCO d'appuyer l'action que mènent les autorités congolaises pour renforcer et réformer les institutions de sécurité et l'appareil judiciaire.

4 MONUSCO, Comprendre le mandat de la MONUSCO. Résolution 1925 du Conseil de sécurité, Octobre 2010, p. 5.

5 Dans les pays post-conflits, comme ici la RDC, il y a le plus souvent un mandat émanant d'une résolution du Conseil de sécurité ou de l'Assemblée générale. Dans des cas précis, notamment dans des situations post-conflits (Kosovo, Liberia, Afghanistan, Sierra Léone, Iraq, Côte d'Ivoire, etc.), le Conseil de sécurité, en coordination avec le Gouvernement concerné, peuvent décider la mise en place une Opération de Maintien de la Paix ou une Mission Politique Spéciale. Composée surtout de militaires et/ou de politiques, ces missions ont le plus souvent une composante électorale.

6 Voir par exemple, la Mission d'observation internationale du Centre Carter en RDC, Elections présidentielle et législatives du 28 Novembre 2011, Déclaration Post - électorale de la compilation et annonce des résultats provisoires de l'élection présidentielle, 10 décembre 2012, « Les résultats provisoires des élections présidentielles annoncées par la Commission électorale nationale indépendante le 9 décembre en République Démocratique du Congo manquent de crédibilité (...) Le processus de compilation à Kinshasa et à Lubumbashi s'est avéré particulièrement problématique. Le manque de préparation évident dans ces deux grandes villes a entraîné de graves irrégularités et a produit un manque de crédibilité dans le processus de compilation. Les déficiences généralisées décrites précédemment se sont déclinées à l'extrême sur ces deux sites » (pp. 1, 4 et 5). 
ne trouverait aucun fondement dans sa Charte. En outre, suite aux contestations des résultats à mi-parcours, à quelles conditions envisager une extension dudit mandat vers une certification internationale du processus par la MONUSCO?

Pour répondre à ces questions, nous jugeons bon d'analyser le bien-fondé de l'octroi d'un tel mandat avant de préciser les contours de son contenu, dans la perspective d'un élargissement de mandat.

\section{Les fondements de l'assistance électorale de la MONUSCO}

Les élections constituent l'un des principaux centres d'intérêt des Nations Unies surtout pour les pays post-conflits. Car, bien conduites, les élections peuvent contribuer à la résolution des conflits à travers la liberté d'expression, le choix par les citoyens des programmes de société et des dirigeants, ainsi que la mise en place d'institutions démocratiques. Par contre, de mauvaises élections peuvent exacerber les conflits et plonger les pays dans le chaos, voire la guerre civile. ${ }^{7}$ De l'entendement du Conseil de sécurité, la tenue en temps voulu d'élections pacifiques, crédibles et transparentes ouvertes à tous, conformément à la Constitution et aux normes internationales, est une condition sine qua non de la consolidation de la démocratie, de la réconciliation nationale et de la restauration d'un climat stable, pacifique et sécurisé dans lequel la stabilisation et la développement socioéconomique du Congo (...)..$^{8}$

Ce point permet d'apprécier le degré d'inadéquation entre les besoins de la RDC et le mandat confié à la Mission des Nations Unies en matière électorale.

7 Ali-Diabacté Tadjoudine, p. 1. Pour cela et de manière générale, plusieurs agences des Nations Unies interviennent dans l'assistance électorale. La répartition des tâches se fait de la façon suivante : le Coordonateur, mandaté par le Résolution 46/37 de mars 1992, approuve ou rejette les requêtes et assure de la coordination et la consistance au sein du système des Nations Unies. La Division de l'Assistance Electorale (DAE) appuie le Coordonateur. Elle étudie les requêtes des Etats membres, conduit des missions d'évaluation des besoins (MEB), maintien un répertoire d'experts électoraux internationaux (Roster), constitue une mémoire institutionnelle en vue de répertorier et de diffuser les bonnes pratiques et les principes internationaux, coordonne les organisations internationales et régionales chargées des élections; les Divisions Régionales du DAP ont en charge l'alerte précoce, la prévention et la résolution des conflits et l'appui (en terme de substance) aux médiations du Secrétaire Général ou de ses représentants, collaborent étroitement avec l'Unité de Soutien de la Médiation (USM). Sur le terrain, plusieurs agences sont impliquées dans la mise en œuvre de la politique électorale approuvée par le Coordonateur. Il s'agit notamment du Département de Maintien de la Paix (DPKO), du Programme des Nations pour le Développement (PNUD), de l'Agence des Nations Unies pour les Opérations Spéciales (UNOPS), et du Bureau du Haut Commissaire aux Droits de l'Homme (OHCR).

8 Rés. 1991 (2011) du Conseil de sécurité des Nations Unies, $7^{\text {m̀me }}$ considérant. 
1. L'assistance électorale et les droits de l'homme dans le système des Nations Unies

L'implication des Nations Unies dans ce domaine tient de manière générale à l'arsenal conventionnel existant et à l'activité du Conseil de sécurité depuis la MONUC, tant le respect de facto des droits de l'homme et des libertés civiques et politiques a été enfreint à plusieurs reprises tout au long du processus électoral.

a. La sauvegarde des droits liés aux élections par l'assistance internationale

Dès le préambule de la Charte des Nations Unies, celles-ci se sont résolues « à proclamer à nouveau leur foi dans les droits fondamentaux de l'homme, dans la dignité et la valeur de la personne humaine, dans l'égalité des droits des hommes et des femmes, ainsi que des nations grandes et petites ». C'est ce que le professeur Mampuya qualifie de substance originelle, la source de tout système qui, à partir des dispositions spécifiques de la Charte, des résolutions et des conventions ultérieures, n'en sera que la mise en ouvre, soit dans ses traits principaux et généraux, soit dans l'une de multiples facettes de cette véritable profession de foi de la Charte. ${ }^{9}$

Dans le corps comme tel de la Charte, d'abord le respect des droits de l'homme figure dans la disposition qui énumère les buts des Nations Unies : « Réaliser la coopération internationale (...) en encourageant le respect des droits de l'homme et les libertés fondamentales pour tous $» .^{10}$

Dans la même lignée s'inscrit la mission confiée à l'Assemblée générale de « provoque(r) des études et fai(re) des recommandations en vue de (...) faciliter pour tous, sans distinction de race, de sexe, de langue ou de religion, la jouissance des droits de l'homme et des libertés fondamentales $»{ }^{11}$ Aussi, les Nations Unies s'assignent-elles la tâche, « en vue de créer les conditions de stabilité et de bien-être nécessaires (...), (de) favoriser le respect universel et effectif des droits de l'homme et des libertés fondamentales pour tous $" .{ }^{12}$ C'est dans ce sens que l'Assemblée générale a donné aux Nations Unies, notamment dans la résolution 62/150 de l'Assemblée générale de mars 2008, le mandat d'apporter une assistance aux Etats membres pour s'assurer que les élections sont conduites périodiquement, de façon pacifique, en conformité avec les normes et les principes internationaux, dans la transparence et dans le respect des libertés fondamentales.

Par ailleurs, la Charte confie à un des organes principaux, le Conseil économique et social, la mission de veiller au respect effectif des droits de l'homme et des libertés fondamentales. Pour ce faire, ce Conseil a reçu un mandat statutaire de créer une commission qui

9 Mampuya Kanunk'a Tshiabo A., " Le système onusien de protection des droits de l'homme : introduction générale », in Droits de l'Homme et Droit International Humanitaire, Séminaire de formation-Cinquantenaire de la DUDH du 18 novembre au 10 décembre 1998, PUK, 1999, p. 29.

10 Article $1^{\text {er }} \S 3$ de la Charte des Nations Unies.

11 Article $13 \S 1^{\text {er }}$, idem.

12 Article $55 \mathrm{c}$, idem. 
serait spécialement chargée de la promotion et de la protection de ces droits de l'homme. Elle fut effectivement créée à la première session dudit Conseil en février 1946, mais remplacée par le Conseil des droits de l'homme. ${ }^{13}$

Il faut signaler que la République Démocratique du Congo applique un monisme avec primauté du droit international. La conséquence en est que divers textes internationaux dont le Pacte relatif aux droits civils et politiques et la Charte africaine des droits de l'homme et des peuples, ratifiés respectivement en 1976 et $1987,{ }^{14}$ font partie intégrante de l'arsenal juridique congolais conformément à l'article 215 de la Constitution. ${ }^{15}$ Celle-ci prend d'ailleurs le soin de rappeler en son sein les libertés fondamentales, notamment les quelques-unes liées directement aux élections.

En effet, les processus électoraux impliquent l'ensemble des droits fondamentaux de l'homme et spécifiquement des droits civils et politiques. Les activités d'assistance et d'observation électorales contribuent donc à promouvoir et à protéger l'ensemble de ces droits. Une élection juste et équitable est une élection qui permet aux différents partis en lice de s'affronter dans un environnement caractérisé par la confiance, la transparence et l'obligation de rendre des comptes. Elle permet aux électeurs de faire un choix informé entre plusieurs alternatives politiques distinctes. Ainsi, un processus électoral véritablement démocratique exige que soient respectés pour l'ensemble des citoyens la liberté d'expression et des médias, les libertés d'association, de réunion et de mouvement, l'Etat de droit, le droit de créer des partis politiques et de se porter candidat à des postes officiels, le droit à la non-discrimination et à l'égalité, le droit de ne pas être la victime de mesures d'intimidation ainsi que tout un ensemble d'autres libertés et droits de l'homme fondamentaux que les Etats se sont engagés à protéger et à promouvoir. ${ }^{16}$

13 Le Conseil des droits de l'homme a été établi par la résolution 60/251 de l'Assemblée générale des Nations Unies du 15 mars 2006.

14 On peut ajouter des textes catégoriels, prévoyant par exemple le droit de participer à des élections libres et régulières, applicable de manière universelle et sans discrimination. L'article 1 de la Convention sur les droits politiques de la femme et l'article 7(a) de la Convention sur l'élimination de toutes les formes de discrimination à l'égard des femmes établissent que les femmes ont le droit de vote dans toutes les élections, dans des conditions d'égalité avec les hommes. Selon l'article 5(c) de la Convention internationale sur l'élimination de toutes les formes de discrimination raciale, "les États parties s'engagent à interdire et à éliminer la discrimination raciale sous toutes ses formes et à garantir le droit de chacun à l'égalité devant la loi sans distinction de race, de couleur ou d'origine nationale ou ethnique, notamment dans la jouissance des droits [...] politiques, notamment droit de participer aux élections - de voter et d'être candidat — selon le système du suffrage universel et égal, droit de prendre part au gouvernement ainsi qu'à la direction des affaires publiques, à tous les échelons, et droit d'accéder, dans des conditions d'égalité, aux fonctions publiques". (Voir également la Déclaration universelle des droits de l'homme, article 2; le Pacte relatif aux droits civils et politiques, article 2.).

15 L'article 215 est ainsi libellé : «Les traités et accords internationaux régulièrement conclus ont, dès leur publication, une autorité supérieure à celle des lois, sous réserve pour chaque traité ou accord, de son application par l'autre partie ».

16 OSCE/BIDDH, Manuel d'observation des élections, Cinquième édition, Varsovie, 2005, p. 11. 
D'abord, la liberté d'expression est essentielle en période électorale dans la mesure où les personnes ne peuvent effectivement exercer leur droit de vote que si elles sont en position de prendre une décision avisée. Le processus électoral est un mécanisme dont l'objet même est l'expression de la volonté politique du peuple. Le droit d'exprimer des idées partisanes doit donc être fermement protégé en période d'élections. ${ }^{17}$ Elle est même essentielle à l'exercice du droit à la liberté de réunion et d'association et du droit de vote. Au-delà de son rappel dans la Constitution et de la précision de son exercice, il est fait obligation à l'Etat congolais de favoriser un environnement médiatique pluraliste. D'où la création en 2011 du Conseil supérieur de l'Audiovisuel, en remplacement de la Haute Autorité des Médias. Toutefois, cet organe n'est pas épargné par la quasi-totalité des rapports établis par divers observateurs, afférents aux atteintes à ce droit.

Ensuite, la liberté d'association et le droit de réunion pacifique sont étroitement liés à la liberté d'expression d'autant plus que restreindre la possibilité de se constituer en association entrave la possibilité de s'exprimer. Cette liberté a une assise conventionnelle internationale, constitutionnelle et légale. Ce droit inclut clairement le droit de constituer des organisations politiques et d'y adhérer. Il est essentiel qu'il soit respecté au cours du processus électoral, puisque la possibilité de constituer des organisations politiques et d'y adhérer est l'un des moyens les plus importants pour la population de participer au processus démocratique.

Quant à la liberté de réunion, pacifique et sans arme, elle sous-entend celle de manifestations pacifiques. Le droit de réunion doit être respecté dans la mesure où les manifestations publiques et les rassemblements politiques font partie intégrante du processus électoral et constituent un mécanisme efficace pour la diffusion de l'information politique. L'expression de ce type de manifestations est soumise à une notification préalable au moins trois jours avant leur tenue en temps normal et vingt-quatre heures durant la campagne électorale.

Il a été rapporté que la liberté de manifestation et d'expression est celle qui a été le plus souvent remise en cause pendant le processus électoral en cours en RDC. Il en est de même du droit à l'intégrité physique et à la vie et du droit à la liberté et à la sécurité. ${ }^{18}$

Concernant le droit à la vie et à l'intégrité physique, ils sont autant garantis par les textes internationaux. Ainsi sont interdits les exécutions extrajudiciaires, sommaires ou arbitraires, la torture et les traitements inhumains et dégradants. Des textes internes congolais reprennent ces exigences, notamment la Constitution et certains législatifs d'application comme le décret $n^{\circ} 002 / 2002$ du 26 janvier 2002 sur l'usage de la force par la Police nationale congolaise. Sur cette base, par exemple, la police ne peut utiliser des armes blanches ou à feu qu'en cas d'extrême nécessité ou, si des policiers veulent disperser la foule à l'aide de ces armes, demander au préalable l'autorisation de l'autorité compétente.

17 Human Rights Library, Manuel de formation sur la surveillance des droits de l'homme, § 14. http:/ /www1.umn.edu/humanrts/monitoring/Fchapter14.html, lu le 24.4.2013.

18 Rapport du BCNUDH, op cit, $\S \S 17-32$. 
Enfin, au sujet du droit à la liberté et à la sécurité de la personne garanti tant par la Pacte international relatif aux droits civils et politiques, la Charte africaine des droits de l'homme et des peuples, la Constitution congolaise que divers autres textes internes, une personne ne peut être arrêtée qu'en respect des procédures établies par la loi. Aussi, toute personne arrêtée doit-elle être informée du motif de son arrestation et traduite dans le plus bref délai devant son juge, sans omettre son droit à entrer en contact avec sa famille ou avec son conseil.

b. Une continuité du mandat électoral de la MONUC à travers la consolidation de la paix

La consolidation de la paix comprend des mesures ciblées visant à réduire les risques de reprise d'un conflit et de jeter les bases d'un développement durable, notamment à travers le renforcement des capacités nationales en matière de gestion des conflits à tous les niveaux. ${ }^{19}$ Ces activités de consolidation de la paix visent ainsi les causes structurelles d'un conflit à travers une approche globale. C'est justement ce que dit le Secrétaire général des Nations Unies pour qui la consolidation de la paix en RDC «consiste, dans un pays au sortir d'un conflit, à rétablir l'autorité de l'Etat, à relancer le dialogue entre les pouvoirs publics et la société civile, à permettre à celle-ci de se régénérer et à établir les assises institutionnelles du développement économique et social $»{ }^{20}$

La situation en RDC présentait déjà ses propres défis, ${ }^{21}$ lesquels avaient amené en son temps le Conseil de sécurité à réorienter le mandat de la MONUC.

En son temps, la Commission Electorale Indépendante (CEI) avait développé une concertation avec les acteurs internationaux que sont en particulier le Comité international d'accompagnement de la Transition (CIAT), la MONUC à travers sa division électorale, le Projet d'appui au processus électoral au Congo (APEC) et la Projet d'appui au cycle électoral (PACE) du PNUD et de l'Union Européenne. Ces structures avaient apporté à la CEI un solide appui technique et logistique. ${ }^{22}$

19 Nations Unies, op cit, p. 19.

20 Rapport du Secrétaire général sur l'activité de la MONUC, 2006, New York, § 18.

21 Certes le conflit armé avait officiellement pris fin en 2003 avec la signature de l'Accord global et inclusif par les parties congolaises, lequel ouvrit la voie à la transition qui conduisit aux élections de 2006. La MONUC était restée au Congo suite à un lourd héritage de la guerre qui aurait fait plus de 4 millions de victimes de 1998 à 2003, doublé d'autres questions récurrentes : l'exploitation des ressources naturelles qui continuent à alimenter des conflits internes, les différends ethniques et fonciers non résolus, l'impact limité du Gouvernement en dehors de Kinshasa, une faible capacité de gouvernance, l'absence des institutions efficaces pour fournir les services de qualité et l'Etat de droit, les violations des droits de l'homme en toute impunité, la corruption endémique, le défi de l'autorité de l'Etat par des rebelles lourdement armés à l'Est, l'intégration des ex rebelles dans les FARDC inachevée et la réforme des services de sécurité nécessaire à l'instauration et au maintien de l'autorité de l'Etat à travers le pays.

22 Pour d'amples détails, lire AfriMAP et OSSISA, op cit, pp. 113-122. 
Ce mandat, encore actuel, consiste en l'appui au Gouvernement en certains secteurs, notamment dans le cadre du Plan de stabilisation et de reconstruction (STAREC) élaboré par le Gouvernement et de la Stratégie internationale d'appui en matière de sécurité et de stabilisation (ISSSS).

Ainsi, la mission de la MONUC dans la préparation et la tenue des élections en République démocratique du Congo avait procédé essentiellement de deux résolutions du Conseil de sécurité : 1496 (2003) du 28 juillet 2003 et 1565 (2004) du $1^{\text {er }}$ août 2004. La tâche était assurée par la Division électorale de la MONUC qui avait reçu pour mandat de fournir une assistance à la CEI.

Dans le cadre des élections de 2006, la MONUC avait aidé dans le renforcement de la capacité institutionnelle de la CEI, l'élaboration et la publication de la feuille de route des élections, dans l'étude de faisabilité concernant le processus électoral, dans la mise au point du calendrier électoral préliminaire, comme dans la détermination du budget des opérations électorales et par un soutien technique afin d'assurer que le cadre législatif pour le processus soit conforme aux standards internationaux et acceptable aussi bien pour la CEI que pour la communauté internationale. ${ }^{23}$ Quant à la sécurisation du processus, elle a planifiée et coordonnée par le Groupe technique de sécurisation électorale, créée en 2005 et qui comprenait des représentants du Gouvernement de transition, de la MONUC, de l'Union Européenne et des autres grands bailleurs de fonds. ${ }^{24}$

La MONUC a participé à la sécurisation du processus en utilisant ses moyens militaires et civils. Elle a aussi renforcé les capacités de la Police nationale congolaise en cette matière. ${ }^{25}$

Toutefois, l'efficacité du rôle de la MONUC dans les élections de 2006 reste contrastée. D'un côté, la Mission a joué déterminant dans l'organisation et la réussite technique du processus. Ses actions majeures sont les conseils et formations dispensées aux responsables et agents de la CEI; la fourniture de l'essentiel de la logistique pour l'acheminement du matériel électoral dans 9000 centres d'inscription, et ensuite dans 50000 bureaux de vote répartis sur l'ensemble du territoire congolais; l'implication directe dans les opérations de rémunération d'environ 260000 agents électoraux et 73000 agents de police qui ont participé à l'organisation et à la sécurité des scrutins. ${ }^{26}$

De l'autre, il a été reproché à la MONUC notamment l'attention proportionnellement grande de cette organisation technique au détriment d'autres tâches autant essentielles pour

23 MONUC, Apercu des rôles..., op cit, p. 12. Lire aussi Mazyambo Makengo Kisala, A., op cit, pp. 176-178.

24 Son rôle et ses réalisations peuvent être lus dans le $20^{\text {ème }}$ Rapport du Secrétaire général des Nations Unies du 28 décembre 2005, § 18.

25 Lire Mazyambo Makengo, op cit, pp. 178-179.

26 . 
la reconstruction de l'Etat pendant la transition : la réforme de l'administration, le renforcement du système judiciaire et la réforme du secteur de sécurité. ${ }^{27}$

Sans pouvoir méconnaître les failles imputables à la MONUC, cette dernière thèse ne nous semble pas suffisamment fondée, à un double point de vue. D'abord, parce que l'on se situe pendant la transition où les élections ont été l'objectif principal. Ensuite le caractère pressant et ponctuel de la tenue des élections de 2006 ne pouvait que pousser le Conseil de sécurité des Nations Unies à privilégier l'appui au processus. Comme l'écrit le Professeur Basue Babu Kazadi : «Force est de souligner que cet état des choses s'explique par la faillite de l'Etat qui, dans un élan pendulaire de liquidation ou de recomposition, a choisi la voie de l'avatar de l'histoire où la carence de légitimité dans la représentation formelle, les élections, est couverte par la légitimité des actes posés principalement en matière de constituante et en matière de gestion de la véritable transition, c'est-à-dire celle qui dans un délai raisonnable et principalement bref, conduit à la tenue des élections libres et honnêtes d'une part et contribue à poser les jalons d'un Etat de droit impulsé par un projet de société démocratique d'autre part ». ${ }^{28}$

C'est dans ce souci de consolider les acquis de la paix que le Conseil de sécurité charge la MONUSCO d'appuyer l'action que mènent les autorités congolaises pour renforcer et réformer les institutions de sécurité et l'appareil judiciaire. ${ }^{29}$ D'où la MONUSCO aidera le Gouvernement de la RDC à renforcer ses capacités militaires, y compris la justice militaire et la police militaire et si, le Gouvernement en fait la demande, aidera à former les bataillons des FARDC et de la police militaire, soutiendra les institutions de justice militaire. La réforme de la police, de la justice et le renforcement de l'autorité de l'Etat sur le territoire libéré des groupes armés sont autant d'actions que doit appuyer la MONUSCO, pour répondre aux besoins exprimés par la RDC en la matière.

2. Les besoins objectifs et la demande formelle de la République Démocratique du Congo en matière électorale

Avec la parenthèse constituée par les élections pluralistes organisées en 1960 et 1965, l'une des crises politiques récurrentes auxquelles la République Démocratique du Congo est confrontée depuis son indépendance reste la contestation de légitimité des institutions due notamment à l'absence d'élections réellement libres et pluralistes. ${ }^{30}$ En dépit d'avancées nota-

27 Austesserre S., « Penser les conflits locaux : l'échec de l'intervention internationale au Congo », in l'Afrique des Grands Lacs, Annuaire 2007-2008, p. 190.

28 Basue Babu Kazadi G., introduction générale à l'étude du droit. Partim Droit public, cours, Faculté de Droit, Université de Kinshasa, 2011-2012, p. 60.

29 Lire MONUSCO, Comprendre le mandat de la MONUSCO. Résolution 1925 du Conseil de sécurité, Octobre 2010, p. 7.

30 AfriMAP et OSISA, République Démocratique du Congo. Démocratie et participation à la vie politique : une évaluation des premiers pas dans la IIIème République, Open Society Foundations, Johannesburg, Novembre 2010, p. 66. 
bles, notamment dans la gestion technique des opérations électorales, les élections de 2006 ont néanmoins été organisées sur une fragile base politique marquée par l'absence d'un consensus solide entre les acteurs politiques et par une dépendance logistique et politique très évidente à l'égard des bailleurs de fonds et des puissances occidentales.

Le cadre juridique qui régit actuellement l'organisation des élections en RDC a été produit dans un contexte post-conflit dans un Etat fragile en refondation, tiraillé entre l'impératif de la démocratisation et la consolidation de la paix que des violences postélectorales risquent de mettre en cause. Les lois électorales reflètent donc davantage les compromis que les anciens belligérants devaient se concéder qu'un effort de permettre la meilleure organisation des élections. ${ }^{31}$

Les besoins objectifs de la RDC en cette matière peuvent se résumer comme suit. Il y a d'abord la nécessité d'instauration d'un climat politique apaisé. Etant donné que la défaillance de l'Etat, les rivalités politiques (différences idéologiques, ethniques, religieuse, raciales), les inégalités socio-économiques et la géopolitique constituent généralement les sources premières des crises sociopolitiques, toutes mesures visant à les résoudre contribuent à la résolution des conflits. Ces dispositions peuvent être prises par la voie législative à travers la Constitution et la loi électorale; ou par des négociations entre les acteurs politiques sous forme d'Accords. Il est de coutume d'amener les forces politiques du pays à adopter des Codes de Conduite relatives aux partis politiques, aux médias, etc. afin de servir de repères éthiques aux citoyens.

Les missions onusiennes, en collaboration avec les équipes pays des Nations Unies contribuent, entre autres, à la « diplomatie préventive » ou à « l'alerte précoce » en vue non seulement de déceler à l'avance les sources potentielles de conflits et de violence, mais surtout d'aider les Etats membres à prendre des mesures adéquates pour les éviter. Les facteurs potentiels de crises liées aux élections sont également mis en lumière par les missions d'évaluation des besoins conduites par la Division de l'Assistance Electorale.

Ensuite, ce pays est à la recherche des conditions sécuritaires propices à l'organisation des élections. En effet, pendant la période électorale dans un pays post-conflit des mesures sécuritaires spéciales sont généralement prises de commun accord par les autorités électorales et les forces de l'ordre. Les Nations Unies contribuent à l'élaboration et à la mise en ouvre de ses mesures à travers les forces de l'ordre de l'ONU dans le cadre des missions de maintien de la paix. Durant les cinq dernières années, la plus grande opération militaire de maintien de la paix ayant soutenu des élections dans un pays post-conflit est la MONUC/ MONUSCO en République Démocratique du Congo avec environs 500 policiers, 17.000 militaires internationaux.

Enfin, le souci de renforcer les capacités de l'administration électorale est un autre besoin non négligeable. D’expérience récente, les élections qui ont débouché sur de vives contestations conduisant à des destructions massives de biens et de propriétés en 2006 et en 2011, entraînant des pertes en vies humaines soulèvent des interrogations profondes sur les 
capacités et les responsabilités de l'organe chargé de leur organisation : la CEI puis la CENI. D'où, pour s'assurer que les élections sont techniquement bien organisées dans un pays post-conflit, les Nations Unies interviennent. Ce qui justifie la plus grande assistance technique de l'ONU de cette nature conduite en RDC, de 2003 à 2007, impliquant plus de 500 experts internationaux, plus de 500 millions de dollars américains et une logistique composée de 68 hélicoptères, 23 avions ainsi que de 5000 véhicules, ${ }^{32}$ tâche qui se poursuit à travers la MONUSCO.

Ces besoins semblent avoir été relativement comblés par l'action des Nations Unies au point que certains soucis récurrents semblent justifier l'élargissement du mandat initial relatif à l'assistance électorale apportée par la MONUSCO.

\section{De l'élargissement du mandat de la MONUSCO vers la certification du processus électoral}

Le rôle de la mission de terrain de l'ONU change selon la nature de son mandat. ${ }^{33} \mathrm{Il}$ est fréquemment demandé aux observateurs de l'ONU et d'autres organisations de jouer plusieurs rôles dans un processus électoral, par exemple aider à préparer les élections, puis les observer.

32 Ali-Diabacté Tadjoudine, op cit, pp 4-5.

33 L'ONU a été impliquée dans des processus électoraux d'au moins quatre manières. La première a consisté à organiser et superviser des élections, dans des pays comme la Namibie. Dans ce cas, l'ONU organise pratiquement tous les aspects du processus électoral. La deuxième est la surveillance des élections, par l'accréditation d'un représentant spécial du Secrétaire général confirmant la validité de certains aspects essentiels du processus électoral. Dans la troisième, le processus électoral est organisé et administré par un organe national, et l'ONU est priée d'observer les élections et de vérifier si le processus s'effectue dans la liberté et la régularité.. Un quatrième type de participation de l'ONU dans l'amélioration des capacités nationales couvre les aspects matériels, infrastructurels, juridiques et relatifs aux droits de l'homme des élections. Les demandes d'assistance technique peuvent être satisfaites rapidement, sans que cette demande ait besoin d'être examinée par un organe directeur de l'ONU (voir Human Rights Library, Manuel de formation sur la surveillance des droits de l'homme, http://wwwl.umn.edu/humanrts/monitoring/Fchapter14.html, lu le 24.4.2013). Dans certains cas rares, les Nations Unies sont elles-mêmes chargées de l'organisation de toutes les opérations électorales. La souveraineté nationale est mise en berne jusqu'à la mise en place des nouvelles institutions issues des élections. Cela a été le cas du Cambodge en 1992-93, du Timor Est en 2001-2002 et du Kosovo de 2000 à 20043. Dans d'autres cas rares, les experts des Nations Unies font partie intégrante de l'administration électorale nationale. La responsabilité est conjointe entre l'Etat membres et l'ONU. C'est le cas de l'Afghanistan en 2004-2005 de l'Irak en 2005 et du Kosovo en 2007. Dans la plupart es cas, les Nations Unies soutiennent, assistent ou accompagnent l'administration électorale nationale, notamment dans les domaines suivants :- Mise en place d'une équipe d'assistance technique composée d'experts internationaux par le PNUD, DPKO ou l'UNOPS, toutes ces agences à travers le répertoire (Roster) de la DAE; - Mobilisation et gestions des ressources des bailleurs de fonds par le PNUD ou l'UNOPS à travers des projets sur base de fonds fiduciaires (Trusts Funds ou Basket Funds); - Coordination des bailleurs de fonds et des organisations internationales impliquées dans le processus électoral par le PNUD ou DPKO; - Mise en place de projets de « cycle électoral » par le PNUD au sein de son programme « Bonne Gouvernance » pour l'assistance technique à moyen et long terme. 
Le besoin du recours à la certification du processus électoral peut se justifier au vu de l'argumentaire ci-dessous, tant il ne s'agit plus seulement d'apprécier les différentes étapes du scrutin comme jusque là assuré par la MONUSCO, mais également de s'assurer que les résultats proclamés reflètent la volonté populaire. Ce dernier volet semble demeurer une impasse pour la consolidation de la paix en RDC.

\section{Un mandat actuel devenu insuffisant}

L'on peut distinguer comme suit cette mission actuelle confiée à la MONUSCO dans ce domaine : l'appui technique et logistique à la CENI et le monitoring des violations des droits de l'homme en rapport avec le processus électoral.

Le paragraphe 7 de la résolution 1991 du Conseil de sécurité prévoit que la MONUSCO prêtera son concours pour l'organisation et la tenue des élections nationales, provinciales et locales, sous forme d'un appui technique et logistique, à la demande des autorités congolaises, en facilitant des échanges élargis et réguliers avec la CENI (...). Cet appui s'exprime en réalité en termes de transport du matériel et de la sécurisation du processus.

Le transport s'entend du déplacement d'une personne ou d'un objet d'un lieu à un autre. Il s'effectue grâce aux moyens tant naturels (jambes, bras, pattes, tête, etc.) qu'artificiels (véhicules, aéronefs, bateaux, téléphones, etc.). Il s'agit ici du déplacement ou déploiement du matériel constitué d'une partie sensible (bulletins de vote) et d'une partie non sensible (kits électoraux, matériel de sensibilisation et formation, isoloirs) depuis les lieux de leur fabrication ou de leur stockage vers différents centres d'utilisation que sont finalement les bureaux de vote.

Les Nations Unies sont surtout actives dans la formation sur la sécurisation des élections. La police des Nations Unies (UNPOL) et leurs Militaires ont souvent pour mandat d'aider l'Etat membre à faire en sorte que tous les agents des forces de sécurité impliqués dans le processus électoral reçoivent une formation appropriée sur les différents aspects des élections, la mission et le comportement des forces de l'ordre, les zones interdites à l'utilisation des armes, le rôle des autorités électorales (logisticien, président du bureau de vote, assesseur).

Le second volet de ce mandat consiste au monitoring des violations des droits de l'homme en rapport avec le processus électoral.

En effet, le concept de monitoring ne figure nulle part dans les résolutions des organes des Nations Unies. Par contre dans le langage officiel, l'on emploi les termes surveillance, contrôle... qui en sont équipollents. En principe, cette surveillance ou ce suivi du processus couvre généralement tout le processus électoral, depuis l'étape préélectorale jusqu'au suivi des résultats proclamés. 
Le Manuel de formation sur le monitoring des droits de l'homme produit par le Haut Commissariat des Nations Unies aux Droits de l'Homme prévoit certains principes ${ }^{34}$ à observer dans la conduite du monitoring des droits de l'homme dans le cadre d'opérations de l'ONU, mais également utile à d'autres contrôleurs des droits de l'homme. Le contrôle s'exerce pendant tout le processus ou concernant uniquement une période considérée relativement au mandat.

La première étape concerne le monitoring des préparatifs pré-électoraux et de la période de campagne. Il s'agit pour l'opération de maintien de la paix de surveiller l'élaboration des lois et procédures électorales, d'assurer le monitoring de l'administration électorale, le monitoring des inscriptions, le monitoring de l'éducation civique, le monitoring des médias, ect.

Ainsi, le 1er novembre 2010 et le 30 septembre 2011, le Bureau Conjoint des Nations Unies aux Droits de l'Homme (BCNUDH) a documenté 188 violations des droits de l'homme apparemment liées au processus électoral. Ces violations portent le plus souvent atteinte à la liberté d'expression des individus, au droit à l'intégrité physique et au droit à la liberté et à la sécurité de la personne, ainsi qu'au droit de réunion pacifique. Des exemples de violences et d'atteintes à l'ordre public par des militants des partis politiques ont également été constatés. ${ }^{35}$

Alors que le rapport ne répertorie pas de manière exhaustive les violations des droits de l'homme et actes de violence, les violations documentées incluent des incidents tels que des menaces de mort contre des défenseurs des droits de l'homme ayant tenu une conférence de presse au cours de laquelle ils ont dénoncé les réformes; des mauvais traitements et arrestations de civils pour le seul port de tee-shirts d'un parti de l'opposition; des convocations répétées à l'Agence nationale de renseignements; des mauvais traitements d'un civil pour avoir posé une question " antipatriotique » et l'arrestation et des mauvais traitements de quatre individus pour avoir eu une discussion politique dans un salon de coiffure.

Bref, le rapport constate que « la préparation des élections a eu lieu dans un climat où les libertés d'expression et d'association étaient limitées ».

34 Ne pas nuire, respecter son mandat, connaître les normes, garder la tête froide, rechercher la consultation, respecter les autorités, la crédibilité, la confidentialité, la sécurité, comprendre le pays, cohérence, persévérance et patience, détail et précision, impartialité, objectivité, la sensibilité, l'intégrité, le professionnalisme et la visibilité (Haut-Commissariat aux droits de l'homme, $\mathrm{Ma}$ nuel de formation sur le monitoring des droits de l'homme, Nations Unies, Genève, S.D., pp. 105-110).

35 Le 1er février 2008, la Division des droits de l'homme (DDH) de la MONUC et le Bureau du Haut- Commissariat aux droits de l'homme (HCDH) en RDC ont fusionné créant ainsi le Bureau Conjoint des Nations Unies aux Droits de l'Homme (BCNUDH). Voir L'ONU tire la sonnette d'alarme sur les violations des droits de l'homme perpétrées pendant la période pré-électorale en RDC, Rapport du BCNUDH sur des violations des droits de l'homme apparemment liées au processus électoral en République démocratique du Congo entre le $1^{\text {er }}$ novembre 2010 et le 30 septembre 2011, Genève/Kinshasa, 9 Novembre 2011. 
Le rapport ajoute qu'« au regard des violences engendrées par les élections de 2006, ainsi que la situation générale particulièrement précaire des droits de l'homme en RDC, les élections de 2011 (présidentielles et parlementaires) constituent un défi majeur pour les droits de l'homme, la sécurité et la consolidation de la démocratie dans le pays ». La plupart des violations perpétrées impliqueraient directement des agents de la Police nationale congolaise ou de l'Agence nationale des renseignements.

D'où dans ce rapport, le BCNUDH demandait instamment au Gouvernement congolais d'intensifier sa coopération avec la société civile, de diffuser des messages publics appelant les agents de l'Etat, particulièrement les membres des forces de sécurité, à promouvoir et respecter les droits de l'homme et à lutter contre l'impunité des agents de l'Etat ayant perpétré des violations des droits de l'homme.

La deuxième étape vise le monitoring du vote comme tel. Il s'agit de s'assurer que l'élection, libre et régulière, est guidée par des dispositions détaillées concernant la forme des bulletins, la conception des urnes et des isoloirs, et la façon de voter. Ces dispositions devraient mettre le processus à l'abri des pratiques frauduleuses tout en respectant le secret du vote; que les bulletins sont présentés clairement et contiennent des informations identiques dans toutes les langues locales, que le bulletin de vote tient compte des divers niveaux d'alphabétisation dans le pays, que le matériel électoral est disponible en quantités suffisantes sur chaque lieu de vote et de tout signe d'intimidation des électeurs ou de traitement discriminatoire des électeurs.

La Mission des Nations Unies ne se mêle pas du processus de scrutin, à moins que son assistance ne soit requise par les autorités. Savoir si elle doit ou non donner suite à de telles demandes d'assistance dépendra des circonstances du moment. En prenant une décision à ce propos, la Mission a à l'esprit son statut d'“observateur", et fera en sorte qu'aucune action entreprise ne puisse en quoi que ce soit être perçu comme partisane, ou mal interprétée de quelque autre façon. Les problèmes sérieux devront être rapportés aux autorités électorales centrales. C'est dans cette logique qu'il convient de comprendre l'attitude passive de la MONUSCO, décriée par certains, relative aux diverses violations des droits de l'homme pendant le déroulement du scrutin qu'elle n'a pas reçu mandat de faire cesser de manière active et de son propre chef.

C'est aussi ici l'occasion d'insinuer le rôle de dénonciateur de telles violations des droits de l'homme, aux dépens, fût-il, du Gouvernement congolais. Au fait, cette mission est traditionnelle aux organisations de défense des droits de l'homme dont le travail consiste à répertorier les cas de violation, à les dénoncer, à prendre contact avec les autorités politico-administratives pour rétablir les victimes dans leurs droits et à sensibiliser et même à mobiliser l'opinion tant nationale qu'internationale. ${ }^{36}$ En pratique, ces dénonciations ne sont pas toujours du goût des autorités congolaises car pouvant contrarier avec la mission primordiale d'appui au Gouvernement congolais lorsque lui sont curieusement imputées droits de l'homme », in Droits de l'Homme et Droit International Humanitaire, op. cit, p. 265. 
nombreuses violations. Les réactions du Gouvernement congolais en réaction au rapport du BCNUDH de décembre 2011 témoignent d'un tel malaise, le qualifiant de partisan, léger, gratuit et incohérent et d'ignorant des missions et du fonctionnement des services de sécurité. ${ }^{37}$

Après les opérations de vote, l'étape suivante est le monitoring du décompte. La mission devrait s'assurer que les processus de comptage des voix, de vérification, de compterendu des résultats, et de conservation des documents officiels, sont sûrs et réguliers; elle devra déterminer si les personnes qui se voient refuser le droit de voter ont accès à une réparation substantielle.

La dernière étape de suivi du processus touche au monitoring des résultats et du suivi. En effet, immédiatement après les élections, les médias demandent habituellement à l'ONU et autres observateurs internationaux de se prononcer quant au caractère libre et régulier de ces élections. Ce n'est qu'après que les plaintes aient été reçues des partis politiques d'opposition, des électeurs et d'autres, que la mission pourra se faire une idée complète des événements. Après les enquêtes sur les plaintes pour déterminer si elles sont fondées, et la précision les faits dénoncés ont pu affecter les résultats, la direction de la mission de droits de l'homme ayant effectué le monitoring des élections sera en mesure d'estimer si celles si ont été libres et régulières ou non. Il faut préciser qu'en aucune circonstance un observateur électoral, ici un membre du personnel de la mission des Nations Unies, ne devra prendre sur lui de s'adresser aux médias pour évaluer ou juger de toute autre manière les élections ou leurs résultats.

Les canaux d'expression de la MONUSCO sont l'appui au Gouvernement congolais et la dénonciation de diverses violations des droits de l'homme, spécialement ceux liées aux élections. C'est dans ce cadre que le Bureau Conjoint des Nations Unies aux Droits de l'Homme publie des rapports dénonçant ces violations. Ce qui parait insuffisant pour garantir la crédibilité du processus au vu des contestations enregistrées concernant des irrégularités avérées, justifiant ainsi un plaidoyer en vue de l'octroi d'un mandat de certification à la Mission.

\section{De la nécessité d'une certification internationale du processus électoral en RDC}

En référence à la pratique des Nations Unies, le mandat de certification avait été confié au Représentant du Secrétaire général des Nations Unies pour la Cote d'Ivoire ${ }^{38}$ chargeant ce dernier de certifier que tous les stades du processus électoral fourniront toutes les garanties nécessaires pour la tenue d'élections présidentielle et législatives ouvertes, libres,

37 Ministère de la Justice et des Droits Humains, Rapport sur la situation des Droits de l'Homme en République du Congo. Droits de l'Homme, élections et questions connexes (Juillet 2011-Février 2012), Kinshasa, mars 2012, pp 31-37.

38 Avant le précédent ivoirien, L'Onu avait déjà accompli des missions de certification des élections dans d'autres pays, mais il s'agissait des élections dont elle avait en charge l'organisation (Timor en 2007 et Népal en 2008). 
justes et transparentes, conformément aux normes internationales. C'est un complément politique, avec l'observation électorale, qui vient légitimer le processus électoral en renforçant sa crédibilité et la confiance des électeurs.

Les arguments qui fondent le recours à ce mécanisme en RDC tiennent surtout à la frustration créée par la composition du Bureau de la CENI, «fort contestée car faisant une fois de plus la part belle à la Majorité Présidentielle et à l'Opposition Parlementaire, au détriment de la Société civile et de l'Opposition extraparlementaire ». Ce manque de confiance dans la composition de l'organe de la gestion des élections connaît une petite histoire en RDC.

Déjà, sous la Conférence Nationale Souveraine (CNS), le problème s'était posé quant à la mise en place de la Commission Nationales des Elections (CNE).$^{39}$ Le professeur Yoka écrit : «Tout le problème est de savoir si la formule conviendra à un peuple qui a fait de la souffrance et de la misère ses grands boucliers contre toute nouvelle forme d'autoritarisme, même si au moment, c'est-à-dire depuis la fin de la CNS, les partis politiques ont euxmêmes prouvé leur fragilité et, pour bon nombre d'entre eux, leur inutilité. Justement, c'est là où il y a nécessité à court terme de la requalification d'une société civile vraiment compétente, engagée, offensive, incorruptible et alternative $\gg .{ }^{40}$

Les événements malheureux qui ont suivi la proclamation des résultats des élections de 2006 et récemment en 2011 sont consécutifs à ce manque de confiance. En effet, après les élections présidentielles et législatives de novembre 2011, de nombreuses missions d'observation électorale avaient critiqué l'organisation de la CENI. Elles avaient estimé pour la plupart d'entre elles que le processus électoral était entaché de beaucoup d'irrégularités et que les résultats des élections n'étaient pas crédibles.

Curieusement, malgré l'urgence de l'amélioration du processus électoral par une profonde restructuration du cadre institutionnel d'organisation des scrutins en RDC, les deux chambres du Parlement congolais ne sont pas parvenues à un compromis autour d'un projet unique de réforme du cadre organique de la CENI. Finalement, un texte controversé a été transmis au Président de la République qui a promulgué le 20 avril 2013 la loi n ${ }^{\circ}$ portant modification de la loi du 28 juillet 2010 créant la CENI. La nouvelle CENI ainsi composée de treize membres et dotée de deux organes, une assemblée plénière et un bureau, ne suscite pas l'unanimité.

Les inquiétudes à ce sujet persistent après cette promulgation tant au lendemain de l'adoption de la nouvelle loi, la Nouvelle Société Civile du Congo avait estimé que malgré les modifications apportées, cette institution restait « politisée à outrance ». L'Union pour la nation congolaise (UNC) parle toujours d'un défaut de représentativité. «Ce n'est pas suffisant, regrette Vital Kamerhe, président de l'UNC, Nous aurions voulu que la CENI soit un

39 Voir la Loi n ${ }^{\circ}$ 95-003 du 10 mai 1995 portant organisation et fonctionnement de la C.N.E., JOZ numéro spécial mai 1995.

40 Yoka Lye Mudaba, «La préparation et la gestion des échéances électorales : Cas de la Commission Nationale des Elections (Cne), in Les défis de la nouvelle République du Congo, Publications des F.C.K, 2003, p. 96. 
organe véritablement neutre, c'est-à-dire que, de par sa composition, elle ne puisse pas favoriser une quelconque force qui va concourir aux élections (...) Il y a un déséquilibre en défaveur de l'opposition et de la société civile ». La Conférence épiscopale nationale du Congo (CENCO) salue bien sûr l'entrée de la société civile dans les instances de la commission, mais regrette qu'elle n'ait pas plus de pouvoir : «La CENI reste encore très politisée, souligne Donatien Nsholé, son secrétaire adjoint. Les attributions accordées par la président [à la société civile, ndlr] nous semblent plus cosmétiques que réelles ».

C'est dans ce dernier point de vue qu'il nous semble situer au fond la vraie justification du manque de confiance aux animateurs de la CENI indistinctement de leurs provenances. Le débat devrait se focaliser plus sur moralité des animateurs de la CENI qu'à celui de leur appartenance politique ou à la société civile. En effet, depuis la CEI, cette institution a toujours formellement été présidée par un membre issu de la société civile. En réalité, ce membre finit toujours par servir les intérêts du parti au pouvoir. Par ailleurs, les membres du Bureau issus de l'Opposition politique n'ont pas toujours été à l'abri, à tort ou à raison, de soupçons de corruption. C'est rappeler le souci de moralisation de l'élite congolaise qui applique en majorité la " politique du ventre ».

C'est dans cet élan et pour pallier à ce manque de confiance que, comme en Côte d'Ivoire, il faut un consensus entre et les acteurs clés nationaux et internationaux concernant l'octroi d'un tel mandat de certification à la MONUSCO,${ }^{41}$ fondée sur les cinq critères principaux suivants: i) un environnement paisible et sécurisé; ii) un processus inclusif, c'est-à-dire sans exclusion; iii) des médias libres et équitables; iv) des listes électorales fiables et; v) des résultats acceptés. ${ }^{42}$ Les trois premiers critères feront l'objet de certification « implicite »; les deux derniers de certification « explicite », par une déclaration formelle du SRSG. ${ }^{43}$ Les listes électorales ne seront déclarées fiables qu'à la suite de consultations dont le but est d'aboutir à un consensus entre les principaux acteurs politiques congolais, la CENI et le Facilitateur à ce sujet.

En effet, la paix et la sécurité constituent le point nodal de la réussite des élections. En ce sens, le Certificateur devait vérifier trois aspects fondamentaux du processus électoral: i) le libre mouvement des populations, y compris des candidats; ii) la liberté d'expression de tous les citoyens; iii) la non-intimidation par les forces de l'ordre, les médias et les autres acteurs. $^{44}$

41 Comme pour le mandat précédent, la première condition d'octroi de ce mandat reste une demande expresse reçue de l'État concerné.

42 Idem.

43 La résolution 1826 adoptée le 29 juillet 2008 par le Conseil de Sécurité des Nations Unies rappelle que « la publication de la liste électorale est une étape cruciale du processus électoral » et « prie le Représentant spécial du Secrétaire général des Nations Unies de certifier cette liste d'une manière explicite ». Il est bien entendu que les résultats seront également certifiés d'une façon expli- cite. C'est une phase du processus tout aussi cruciale que celle de la liste des électeurs.

44 Idem. 
Un deuxième atout réside au caractère contraignant de la certification, contrairement aux autres mécanismes classiques de contrôle international des élections, tel le mandat actuel de la MONUSCO qualifié d'insuffisant. En effet, les résultats ainsi certifiés s'imposeraient à tous les acteurs du processus au point qu'aucune contestation non démocratique ou compromission ne seraient admises après la certification des résultats, les parties étant censées se soumettre aux décisions du certificateur.

Un autre avantage de la certification du processus électoral tient à ce qu'il vise à garantir la conformité de toutes les étapes dudit processus aux normes internationales.

Toutefois, l'octroi d'un tel mandat de certification du processus électoral à la MONUS$\mathrm{CO}$, malgré son efficacité prouvée ailleurs, devait relever certains défís liés tant à son matérialisation que dans sa mise en œuvre. En effet, l'absence de consensus national en vue de la formalisation d'une demande officielle par le gouvernement de la RDC nous semble le premier écueil. Or cela nécessite une volonté et l'engagement politiques des gouvernements et de la société civile congolaise. L'on ne saurait minimiser le soutien requis de la communauté internationale.

Dans sa mise en œuvre, la certification souhaitée du processus électoral en RDC, désormais partielle, étant donné que les élections constituant la première frange de novembre 2012 n'ont pas été certifiées, buterait au manque de diagnostic complet des besoins réels de ce pays. Elle se limiterait aux seules et prochaines élections provinciales, municipales, urbaines et locales. Le risque de contamination mérite d'être suffisamment pris en compte pour l'éviter.

L'on ne saurait enfin ignorer le fait qu'il est difficile aux personnes mêmes, ici les différents partenaires dont la Mission des Nations Unies, ayant contribué à préparer et à soutenir un processus électoral de demeurer parfaitement impartiales dans l'observation des résultats de leurs propres efforts. Mais ce souci semble couvert par le caractère contraignant de la certification.

\section{Conclusion}

La certification qui est un mandat récent vise à suppléer la crise de confiance chronique des populations et/ou des acteurs vis-à-vis des institutions en charge de l'organisation et de la conduite des processus électoraux dans des pays en crise. Comme tout instrument, la certification est un couteau à double tranchant. D'un côté, sa mise à œuvre est d'autant plus difficile que son contenu reste à préciser selon le contexte sociopolitique de chaque pays concerné. La certification pourrait être mal vécue par la partie nationale si le certificateur est perçu comme un « proconsul ». ${ }^{45}$

D'un autre côté, utilisée à bon escient, la certification pourrait contribuer efficacement à la prévention et à la gestion des conflits liés aux élections dans ce pays à démocratie naissante, la RDC. 


\section{Références bibliographiques}

AfriMAP et OSISA, République Démocratique du Congo. Démocratie et participation à la vie politique : une évaluation des premiers pas dans la IIIème République, Open Society Foundations, Johannesburg, Novembre 2010.

Ali-Diabacté Tadjoudine, " La problématique des élections après un conflit et de la certification », Séminaire de l'Organisation Internationale de la Francophonie, Nations Unies, New York, 11 décembre 2008.

Austesserre S., « Penser les conflits locaux : l'échec de l'intervention internationale au Congo », in L'Afrique des Grands Lacs, Annuaire 2007-2008, p. 190.

Basue Babu Kazadi G., Introduction générale à l'étude du droit. Partim Droit public, cours, Faculté de Droit, Université de Kinshasa, 2011-2012.

Claire Moucharafieh, « Pas d'opérations internationales de maintien de la paix sans respect des droits de l'homme », http://base.d-p-h.info/fr/fiches/premierdph/fiche-premierdph-1524.html, lu le 24.4.2013.

Droits de l'Homme et Droit International Humanitaire, Séminaire de formation-Cinquantenaire de la DUDH du 18 novembre au 10 décembre 1998, PUK, 1999.

Évariste Sonon, « Assistance électorale », Réseau de recherche sur les opérations de paix, 28 juillet 2012, http://www.operationspaix.net/12-resources/details-lex ique/assistance-electorale.htmlHaut-Commissariat aux droits de l'homme, Manuel de formation sur le monitoring des droits de l'homme, Nations Unies, Genève, S.D.

Human Rights Library, Manuel de formation sur la surveillance des droits de l'homme, http://www 1.umn.edu/humanrts/monitoring/Fchapter14.html, lu le 24.4.2013.

Loi n 95-003 du 10 mai 1995 portant organisation et fonctionnement de la C.N.E., JOZ numéro spécial mai 1995.

Ministère de la Justice et des Droits Humains, Rapport sur la situation des droits de l'Homme en République du Congo. Droits de l'Homme, élections et questions connexes (Juillet 2011-Février 2012), Kinshasa, mars 2012,

Mission d'observation internationale du Centre Carter en RDC, Elections présidentielle et législatives du 28 Novembre 2011, Déclaration Post - électorale de la compilation et annonce des résultats provisoires de l'élection présidentielle, 10 décembre 2012.

Mission d'observation électorale de l'Union Européenne en République Démocratique du Congo, Une forte mobilisation de l'électorat dans un processus insuffisamment maîtrisé, Rapport préliminaire, Kinshasa, 1er décembre 2012.

MONUSCO, Comprendre le mandat de la MONUSCO. Résolution 1925 du Conseil de sécurité, Kinshasa, octobre 2010.

OSCE/BIDDH, Manuel d'observation des élections, Varsovie, 2005.

Rapport d'enquête du Bureau Conjoint des Nations Unies aux Droits de l'Homme sur les violations graves des droits de l'homme commises par des membres des forces de défense et de sécurité congolaises dans la ville de Kinshasa en République Démocratique du Congo, MONUSCO-Nations Unies, mars 2012. 
Rapport du Secrétaire général sur l'activité de la MONUC, 2006.

Résolution 1925 (2010) du Conseil de sécurité des Nations Unies du 28 mai 2010.

Yoka Lye Mudaba, « La préparation et la gestion des échéances électorales : Cas de la Commission Nationale des Elections (Cne), in Les défis de la nouvelle République du Congo, Publications des F.C.K, 2003, pp. 86-96. 